\title{
TWO-PEAK SOLITON IN THE CKP HIERARCHY
}

\author{
JINGSONG HE†‡, YI CHENG†AND RUDOLF A. RÖMER
}

$\dagger$ Department of Mathematics, University of Science and Technology of China, Hefei, 230026 Anhui, P.R. China

$\ddagger$ Department of Physics and Centre for Scientific Computing, University of Warwick, Coventry CV4 7AL, United Kingdom

\begin{abstract}
We present a systematic approach to the construction of soliton solutions for the 5reduction of the C-type sub-hierarchy for the Kadomtsev-Petviashvili(CKP) hierarchies starting from the general $\tau$-function $\tau^{(n+k)}$ of the Kadomtsev-Petviashvili (KP) hierarchy. We obtain the one-soliton and two-soliton solutions for the bi-directional Kaup-Kupershmidt (bKK) equation, i.e. the 5-reduction of CKP hierarchy.
\end{abstract}

\section{INTRODUCTION}

The bi-directional Kaup-Kupershmidt (bKK) equation [1,2],

$$
\left(z_{x x x x x}+15 z_{x} z_{x x x}+15 z_{x}^{3}-15 z_{x} z_{t}-5 z_{x x t}+\frac{45}{4} z_{x x}^{2}\right)_{x}-5 z_{t t}=0 .
$$

is introduced by an "exchange procedure" as the bi-directional analogue of the Kaup-Kupershmidt (KK) equation. More precisely, bKK is the 5-reduction of the C-type sub-hierarchy of the KadomtsevPetviashvili (CKP) hierarchy. Its Lax pair is $[1,2]$

$$
\begin{gathered}
{\left[\partial_{x}^{5}+5 u \partial_{x}^{3}+\frac{15}{2} u_{x} \partial_{x}^{2}+\left(5 u^{2}+\frac{35}{6} u_{x x}+\frac{5}{3} z_{t}\right) \partial_{x}+5 u u_{x}+\frac{5}{3} u_{x x x}+\frac{5}{6} u_{t}\right] \phi=\lambda \phi,} \\
\partial_{t} \phi=\left(\partial_{x}^{3}+3 u \partial_{x}+\frac{3}{2} u_{x}\right) \phi, \quad u=z_{x}, t_{3}=t, t_{1}=x,
\end{gathered}
$$

Here $u=u_{2}$ comes from the Lax operator $L$ of CKP $[3,4]$

$$
L=\partial+u_{2} \partial^{-1}+u_{3} \partial^{-2}+\cdots, \quad L^{*}=-L,
$$

and the conjugation operation "*" is defined by $\partial^{*}=-\partial,(A \circ B)^{*}=B^{*} \circ A^{*}$.

An essential characteristic of the KP hierarchy is the existence of the $\tau$-function and all dynamical variables $\left\{u_{i}\right\}, i=2,3, \ldots$, can be constructed from it [3,4]; e.g., $u_{2}=\frac{\partial^{2}}{\partial x^{2}} \log \tau, u_{3}=$ $\frac{1}{2}\left(-\frac{\partial^{3}}{\partial x^{3}}+\frac{\partial^{2}}{\partial x \partial t_{2}}\right) \log \tau, \ldots$. Thus the construction of the $\tau$-function is the central task in order to obtain the dynamical variables $\left\{u_{i}\right\}$ associated with the KP hierarchy.

Gauge transformations [5,6] offer an efficient route towards this goal. In Ref. [7] two kinds of useful gauge transformations have been proposed, namely,

$$
T_{D}(\phi)=\phi \partial \phi^{-1}, \quad \text { and } \quad T_{I}(\psi)=\psi^{-1} \partial^{-1} \psi
$$

resulting in a very general and universal $\tau$-function (see equation (3.17) of [7] and also $I W_{k, n}$ in [8]). The eigenfunction $\phi$ and the adjoint eigenfunction $\psi$ of the KP hierarchy are defined by

$$
\frac{\partial \phi}{\partial t_{n}}=B_{n} \phi, \quad \frac{\partial \psi}{\partial t_{n}}=-B_{n}^{*} \psi
$$

where $\phi=\phi(\lambda ; \bar{t})$ and $\psi=\psi(\lambda ; \bar{t})$ and $\bar{t}=\left(t_{1}, t_{2}, \cdots\right)$.

Date: Revision : 2.1, compiled October 10, 2005. 
There are two steps that arise when one wants to study the solutions of the $(1+1)$-dimensional soliton equations given by the $n$-reduction of the CKP hierarchies. The first is how it retain the restrictions, i.e. $L^{*}=-L$ for CKP, for the transformed Lax operators $L^{(1)}=T L T^{-1}$. In other words, the problem is how to obtain the $\tau$-functions $\tau_{\text {CKP }}^{(n+k)}$ from the general $\tau$-function $\tau^{(n+k)}=I W_{k, n} \tau^{(0)}$ with the gauge transformation $T_{n+k}$ of the KP hierarchy. Here $\tau^{(0)}$ is the initial value of the $\tau$-function of the KP hierarchy. Also, the generating functions $\phi_{i}, \psi_{i}$ of the gauge transformation will be complexvalued and related to the $n$-th roots of $e^{i \varepsilon}$. The second issue therefore is how to choose generating functions $\phi_{i}=\phi\left(\lambda_{i} ; x, t\right)$ and $\left.\psi_{i}=\psi\left(\mu_{i} ; x, t\right)\right)$ such that $\tau_{\text {CKP }}^{(n+k)}$ correspond to a physical $\tau$-function $\hat{\tau}_{E q}^{(n+k)}$, namely such that it is real and positive on the full $(x, t)$ plane. Here we shall outline how do to this for the 5-reduction, i.e. the bKK equation.

\section{2. $\tau$-FUNCTION FOR CKP HIERARCHY}

The starting point for the solution of the bKK equation is the $\tau$-function of the KP hierarchy.

Lemma 2.1 ( $[7,8])$. The $\tau$-function of the KP hierarchy generated by the gauge transformation $T_{n+k}$ is given as

$$
\tau^{(n+k)}=I W_{k, n}\left(\psi_{k}^{(0)}, \psi_{k-1}^{(0)}, \cdots, \psi_{1}^{(0)} ; \phi_{1}^{(0)}, \phi_{2}^{(0)}, \cdots, \phi_{n}^{(0)}\right) \tau^{(0)},
$$

where $\left(\phi_{i}^{(0)}, \psi_{j}^{(0)}\right)=\left(\phi\left(\lambda_{i} ; \bar{t}\right), \psi\left(\mu_{j} ; \bar{t}\right)\right)$ are solutions of equation (6) with initial value $\tau^{(0)}$ for the $\tau$-function and the initial values of the $\left\{u_{i}\right\}$ are $\left\{u_{i}^{(0)}\right\}$. Here $\left\{\phi_{i}^{(0)}, \psi_{j}^{(0)}\right\}$ are called generating functions(GFs) of gauge transformation.

Let us now discuss how to reduce $\tau^{(n+k)}$ in (7) to the $\tau$-function of the CKP hierarchy. The key problem is how to retain the restriction $\left(L^{(n+k)}\right)^{*}=-L^{(n+k)}$ under the gauge transformation $T_{n+k}[8]$. We note that $\bar{t}=\left(t_{1}, t_{3}, t_{5}, \cdots\right)$ in CKP hierarchy.

Proposition 2.1 (see Refs. [9,10]).

(1) The appropriate gauge transformation $T_{n+k}$ is given by $n=k$ and generating functions $\psi_{i}^{(0)}=$ $\phi_{i}^{(0)}$ for $i=1,2, \ldots, n$.

(2) The $\tau$-function $\tau_{\mathrm{CKP}}^{(n+n)}$ of the CKP hierarchy has the form

$$
\tau_{\text {CKP }}^{(n+n)}=I W_{n, n}\left(\phi_{n}^{(0)}, \phi_{n-1}^{(0)}, \cdots, \phi_{1}^{(0)} ; \phi_{1}^{(0)}, \phi_{2}^{(0)}, \cdots, \phi_{n}^{(0)}\right) \tau_{\text {CKP }}^{(0)}
$$

If the initial values of the dynamical variables $\left\{u_{i}\right\}$ of the CKP hierarchy are zero, then $\tau_{\text {CKP }}^{(0)}=1$ and the equations in $(6)$ of $\left(\phi_{i}^{(0)}, \psi_{j}^{(0)}\right)=\left(\phi\left(\lambda_{i} ; \bar{t}\right), \psi\left(\mu_{j} ; \bar{t}\right)\right)$ become more simpler as

$$
\frac{\partial \phi(\lambda ; \bar{t})}{\partial t_{n}}=\left(\partial_{x}^{n} \phi(\lambda ; \bar{t})\right), \quad \frac{\partial \psi(\mu ; \bar{t})}{\partial t_{n}}=(-1)^{n+1}\left(\partial_{x}^{n} \psi(\mu ; \bar{t})\right)
$$

\section{SOLITON SOLUTIONS OF BKK EQUATION}

The 5-reduction of the CKP hierarchy yields the bKK equation equation (1). Let the initial value be $u=0$ in Eqs. (2) and (3), then $\phi_{i}^{(0)}=\phi\left(\lambda_{i} ; x, t\right)$ are solutions of

$$
\partial_{x}^{5} \phi\left(\lambda_{i} ; x, t\right)=\lambda_{i} \phi\left(\lambda_{i} ; x, t\right), \quad \frac{\partial \phi\left(\lambda_{i} ; x, t\right)}{\partial t}=\left(\partial_{x}^{3} \phi\left(\lambda_{i} ; x, t\right)\right) .
$$

So Proposition 2.1 implies the $\tau$-function of the bKK equation.

Proposition 3.1. The $\tau$-function of the bKK equation generated by $T_{n+n}$ from initial value 1 is

$$
\tau_{\text {bKK }}^{(n+n)}=I W_{n, n}\left(\phi_{n}^{(0)}, \phi_{n-1}^{(0)}, \cdots, \phi_{1}^{(0)} ; \phi_{1}^{(0)}, \phi_{2}^{(0)}, \cdots, \phi_{n}^{(0)}\right)
$$


and the solution $u$ of the bKK from initial value zero is

$$
u=\left(\partial_{x}^{2} \log \tau_{\mathrm{bKK}}^{(n+n)}\right) .
$$

Here $\phi_{i}^{(0)}=\phi\left(\lambda_{i} ; x, t\right)$ are solutions of equation (10).

In general, this $\tau$-function $\tau_{\mathrm{bKK}}^{(n+n)}$ for bKK is complex and related to the 5 -th roots of $e^{i \varepsilon}$. We have to find the real and non-zero $\tau$-function from it such that $u$ in equation (12) is a real and smooth solution of bKK. This is the main task of this section. We start by analysing the solution $\phi(\lambda ; x, t)$ of equation (10) and make the general ansatz

$$
\phi(\lambda ; x, t)=\sum_{j=1}^{5} A_{j} e^{x p_{j}+t p_{j}^{3}}, \quad \text { with } \quad p_{j}^{5}=\lambda .
$$

Here $p_{j}=k \exp \left(\frac{\varepsilon+2 \pi j}{5} i\right), k^{5}=|\lambda|, k \in \mathbb{R}, 0 \leq \varepsilon<2 \pi$ and $j=0,1,2,3,4$. There are two important ingredients which we can use to find the desired solution. The first is that the 5 -th roots $\varepsilon_{j}=$ $\exp \left(\frac{\varepsilon+2 \pi j}{5} i\right)$ of $e^{i \varepsilon}$ are distributed uniformly on the unit circle in $\mathbb{C}$. So for a suitable value of $\varepsilon$ there exist combinations of $p_{j}$ 's which are symmetric upon reflection on the $x$-axes; similarly for the $y$-axes for other values of $\varepsilon$. The second ingredient is that $\tau_{\mathrm{bKK}}$ and $\exp (\alpha x+\beta t) \tau_{\mathrm{bKK}}$ will imply the same solution $u$ since $u=\partial_{x}^{2} \log \tau_{\mathrm{bKK}}$. Here, $\alpha$ and $\beta$ are arbitrary complex constants. Therefore we can obtain the desired real and smooth solutions of the bKK if $\tau_{\text {bKK }}$ can be expressed as $\tau_{\mathrm{bKK}}=e^{\alpha x+\beta t} \hat{\tau}_{\mathrm{bKK}} \approx \hat{\tau}_{\mathrm{bKK}}$. Here, $\hat{\tau}_{\mathrm{bKK}}$ is a real and nonzero function although $\tau_{\mathrm{bKK}}$ is complex. We call $\hat{\tau}_{\mathrm{bKK}}$ the physical $\tau$-function for the bKK equation. Based on the above arguments, let us make the refined ansatz

$$
\phi\left(\lambda_{1} ; x, t\right)=A_{1} e^{p_{1} x+p_{1}^{3} t}+B_{1} e^{q_{1} x+q_{1}^{3} t}, \quad p_{1}=k_{1} e^{i \varepsilon_{1}}, q_{1}=-k_{1} e^{-i \varepsilon_{1}}, k_{1}^{5}=\left|\lambda_{1}\right|, k_{1} \in \mathbb{R},
$$

or

$$
\phi\left(\lambda_{1} ; x, t\right)=A_{1} e^{p_{1} x+p_{1}^{3} t}+B_{1} e^{q_{1} x+q_{1}^{3} t}, \quad p_{1}=k_{1} e^{i \varepsilon_{1}}, q_{1}=k_{1} e^{-i \varepsilon_{1}}, k_{1}^{5}=\left|\lambda_{1}\right|, k_{1} \in \mathbb{R},
$$

and we next need to fix the ratio $\frac{B_{1}}{A_{1}}$. In order to do so, let us study a simple case which is generated by the gauge transformations $T_{1+1}$.

Proposition 3.2. Let $\xi_{1}=x k_{1} \cos \varepsilon_{1}+t k_{1}^{3} \cos 3 \varepsilon_{1}, \frac{B_{1}}{A_{1}}=i e^{-i \varepsilon_{1}}$ and $\phi_{1}^{(0)}=\phi\left(\lambda_{1} ; x, t\right)$ defined by equation (14), then $\hat{\tau}_{\mathrm{bKK}}^{(1+1)}=e^{2 \xi_{1}}+e^{-2 \xi_{1}}+\frac{2}{\sin \varepsilon_{1}}$ is extracted from $\left.\tau_{\mathrm{bKK}}^{(n+n)}\right|_{n=1}$, and one soliton solution is $u=\left(\partial_{x}^{2} \log \hat{\tau}_{\mathrm{bKK}}^{(1+1)}\right)=\frac{4 k_{1}^{2}\left(\cos \varepsilon_{1}\right)^{2}\left(1+\frac{\cosh 2 \xi_{1}}{\sin \varepsilon_{1}}\right)}{\left(\cosh 2 \xi_{1}+\frac{1}{\sin \varepsilon_{1}}\right)^{2}}$ with $\varepsilon_{1}=\frac{\pi}{10}$ or $\frac{3 \pi}{10}$. The velocity of the soliton is $v=-k_{1}^{2} \frac{\cos 3 \varepsilon_{1}}{\cos \varepsilon_{1}}$. In particular, the left-going soliton have two peaks in its profile and the negative speed $v_{-}=\left.v\right|_{\varepsilon_{1}=\frac{\pi}{10}}$; the right-going soliton have only one peak and positive speed $v_{+}=\left.v\right|_{\varepsilon_{1}=\frac{3 \pi}{10}}$.

Remark 3.1. If $\phi_{1}^{(0)}=\phi\left(\lambda_{1} ; x, t\right)$ as defined by equation (15), we can also get periodic solutions of the bKK equations. We omit this kind of solution for brevity.

Now we turn to the more complicated $\tau_{\text {bKK }}^{(2+2)}$ from Proposition 3.1, which generates the two soliton of the bKK equation. 
Proposition 3.3. There exist suitable $\frac{B_{i}}{A_{i}}(i=1,2)$ such that the physical $\tau$-function of bKK $\hat{\tau}_{\mathrm{bKK}}^{(2+2)}$ can be expressed as

$$
\begin{aligned}
& \hat{\tau}_{\mathrm{bKK}}^{(2+2)}= \\
& \left\{\frac{e^{2\left(\xi_{1}+\xi_{2}\right)}}{4 k_{1} k_{2}\left(k_{1}^{2}+k_{2}^{2}+2 k_{1} k_{2} \cos \left(\varepsilon_{1}-\varepsilon_{2}\right)\right)^{2}}+\frac{g_{9} e^{-2\left(\xi_{1}+\xi_{2}\right)}}{4 k_{1} k_{2}\left(k_{1}^{2}+k_{2}^{2}+2 k_{1} k_{2} \cos \left(\varepsilon_{1}-\varepsilon_{2}\right)\right)^{2}}\right. \\
& +\frac{e^{2\left(\xi_{1}-\xi_{2}\right)}}{4 k_{1} k_{2}\left(k_{1}^{2}+k_{2}^{2}-2 k_{1} k_{2} \cos \left(\varepsilon_{1}+\varepsilon_{2}\right)\right)^{2}}+\frac{e^{-\left(2 \xi_{1}-\xi_{2}\right)}}{4 k_{1} k_{2}\left(k_{1}^{2}+k_{2}^{2}-2 k_{1} k_{2} \cos \left(\varepsilon_{1}+\varepsilon_{2}\right)\right)^{2}} \\
& +\frac{e^{2 \xi_{1}}}{2 k_{1} k_{2} \sin \varepsilon_{2}\left(k_{1}^{2}+k_{2}^{2}+2 k_{1} k_{2} \cos \left(\varepsilon_{1}-\varepsilon_{2}\right)\right)\left(k_{1}^{2}+k_{2}^{2}-2 k_{1} k_{2} \cos \left(\varepsilon_{1}+\varepsilon_{2}\right)\right)} \\
& +\frac{g_{8} e^{-2 \xi_{1}}}{2 k_{1} k_{2} \sin \varepsilon_{2}\left(k_{1}^{2}+k_{2}^{2}+2 k_{1} k_{2} \cos \left(\varepsilon_{1}-\varepsilon_{2}\right)\right)\left(k_{1}^{2}+k_{2}^{2}-2 k_{1} k_{2} \cos \left(\varepsilon_{1}+\varepsilon_{2}\right)\right)} \\
& +\frac{e^{2 \xi_{2}}}{2 k_{1} k_{2} \sin \varepsilon_{1}\left(k_{1}^{2}+k_{2}^{2}+2 k_{1} k_{2} \cos \left(\varepsilon_{1}-\varepsilon_{2}\right)\right)\left(k_{1}^{2}+k_{2}^{2}-2 k_{1} k_{2} \cos \left(\varepsilon_{1}+\varepsilon_{2}\right)\right)} \\
& +\frac{g_{6} e^{-2 \xi_{2}}}{2 k_{1} k_{2} \sin \varepsilon_{2}\left(k_{1}^{2}+k_{2}^{2}+2 k_{1} k_{2} \cos \left(\varepsilon_{1}-\varepsilon_{2}\right)\right)\left(k_{1}^{2}+k_{2}^{2}-2 k_{1} k_{2} \cos \left(\varepsilon_{1}+\varepsilon_{2}\right)\right)} \\
& \left.+\frac{g_{5}\left(\left(k_{1}^{2}+k_{2}^{2}\right)^{2}-4 k_{1}^{2} k_{2}^{2}\left(\cos ^{2} \varepsilon_{1} \cos ^{2} \varepsilon_{2}+\sin ^{2} \varepsilon_{1} \sin ^{2} \varepsilon_{2}\right)\right)}{k_{1} k_{2} \sin \varepsilon_{1} \sin \varepsilon_{2}\left(k_{1}^{2}+k_{2}^{2}+2 k_{1} k_{2} \cos \left(\varepsilon_{1}-\varepsilon_{2}\right)\right)\left(k_{1}^{2}+k_{2}^{2}-2 k_{1} k_{2} \cos \left(\varepsilon_{1}+\varepsilon_{2}\right)\right)}\right\}
\end{aligned}
$$

The two solitons solution is $u=\left(\partial_{x}^{2} \log \hat{\tau}_{\mathrm{bKK}}^{(2+2)}\right)$. In particular, $\varepsilon_{1}=\varepsilon_{2}=\frac{\pi}{10}$ results in two overtaking soltions moving in negative direction; $\varepsilon_{1}=\varepsilon_{2}=\frac{3 \pi}{10}$ results in two overtaking soltions moving in positive direction; $\varepsilon_{1}=\frac{\pi}{10}, \varepsilon_{2}=\frac{3 \pi}{10}$ results in head-on colliding two soltions.

\section{Conclusions and Discussions}

Our construction of the $\tau$-function for the bKK equation can be summarized as follows: $\tau^{(n+k)}$ $\left.\stackrel{\text { constraints of GFs and } k=n}{\longrightarrow} \tau_{\mathrm{CKP}}^{(n+k)} \stackrel{5-\text { reduction }}{\longrightarrow} \tau_{\mathrm{bKK}}^{(n+n)} \stackrel{\text { suitable } \frac{B_{i}}{A_{i}}}{\longrightarrow} \hat{\tau}_{\mathrm{bKK}}^{(n+n)}\right|_{n=1,2}$. Based on this method, we have obtained the one and two soliton solutions of the bKK equation. These solutions are consistent with the results of $[1,2,11]$, in which the Hirota method and Bäcklund transformations have been used. Our method appears to be more generally applicable and transparent from the point of view of the KP hierarchy. Moreover, it can be applied to higher-order reductions of CKP and to the B-type sub-hierarchy of the KP hierarchy (BKP).

This work was supported partly by project 973 "Nonlinear Science" and the National Natural Science Foundation of China (10301030). Support from the Warwick Chinese Fellowship Funds is grateful acknowledged.

\section{REFERENCES}

[1] J. M. Dye, A.Parker, On bidirectional fifth-order nonlinear evolution equations, Lax pairs, and directionally solitary waves. J. Math. Phys. 42, 2567-2589(2001)

[2] J. M. Dye, A. Parker, A bidirectional Kaup-Kupershmidt equation and directionally dependent solitons. J. Math. Phys. 43, 4921-4949(2002)

[3] E. Date, M. Kashiwara, M. Jimbo, T. Miwa, in Nonlinear Integrable Systems- Classical and Quantum Theory, edited by M. Jimbo and T. Miwa (World Scientific, Singapore, 1983) p. 39-119.

[4] L. A. Dickey, Soliton Equations and Hamiltonian Systems (World Scintific, Singapore, 1991).

[5] V. E. Zakharov, A. V. Mikhailov, Relativistically invariant two-dimensional models of field theory which are integrable by means of the inverse sacttering problem method. Sov. Phys. JETP47,1017-1027(1978).

[6] V. B. Matveev, M. A. Salle, Darboux Transformations and Solitons (Springer-Verlag, Berlin, 1991).

[7] L. L. Chau, J. C. Shaw, H. C. Yen, Solving the KP hierarchy by gauge transformations. Commun. Math. Phys.149, 263-278(1992). 
[8] J. S. He, Y. S. Li, Y. Cheng, The determinant representation of the gauge transformation operators. Chin. Ann. of Math.23B, 475-486(2002).

[9] J. J. Nimmo, "Darboux transformation from reduction of the KP hierarchy ", in Nonlinear Evolution equation and Dynamical Systems, edited by V. G. Makhankov et al(Singapore: World Scientific, 1995)P168-177.

[10] A. Mei, Darboux Transformations for Antisymmetric Opertor and BKP Integrable Hierarchy(in Chinese). Master Thesis, University of Science and Tehnology of China(1999).

[11] C. Verhoeven, M. Musette, Soliton solutions of two bidirectional sixth-order partial differential equations belonging to the KP hierarchy. J. Phys. A 36, L133-L143(2003) 